UTPT-94-20

hep-ph/9407311

\title{
Hints of new flavor physics at LEP?
}

\author{
B. Holdom币 \\ Department of Physics \\ University of Toronto \\ Toronto, Ontario \\ Canada M5S $1 A 7$
}

\begin{abstract}
A massive gauge boson coupling to the third family but not lighter families mixes with the $Z$. The $Z$ partial width to $b \bar{b}$ increases, the $\tau$ asymmetry parameter increases, the invisible width of the $Z$ decreases, and $\alpha_{s}\left(m_{Z}\right)$ decreases, all in a manner consistent with present data.
\end{abstract}

\footnotetext{
${ }^{1}$ holdom@utcc.utoronto.ca
} 
The large top mass may signal the presence of new gauge symmetries which are broken close to the $1 \mathrm{TeV}$ energy scale, and these gauge interactions may also be felt by other members of the third family. For example there have been analyses of the effects of extended technicolor (ETC) interactions on the $Z b \bar{b}$ vertex by relating these effects to the physics responsible for the top mass. [1] This is the physics of technicolored ETC gauge bosons which cause transitions between technifermions and the third family.

In this note we would like to consider the effects of a gauge boson which is a singlet under unbroken gauge symmetries and with a mass in the few hundred $\mathrm{GeV}$ to one $\mathrm{TeV}$ range. Such a gauge boson, $X$, may correspond to a diagonal generator of some broken flavor gauge symmetry. The important point is that it is typical for the last flavor symmetry to break to involve the heaviest family, and thus the $X$ boson naturally couples to the third family but not lighter families. Its main effects may arise through a small mixing with the $Z$. These effects will differ from those of the more often studied $Z^{\prime}$ appearing in the grand unified context, since the $Z^{\prime}$ couples universally to all families of fermions.

An extra gauge boson can mix with the $Z$ through a mass mixing term and a kinetic energy mixing term. It can also mix with the photon through a kinetic energy mixing. These mixings show up in shifts in the standard electroweak correction parameters, $S, T$, and $U$. And since the mixing induces small $X$ couplings to the light families proportional to the $Z$ and photon couplings, the standard neutral current processes are also affected by $X$ exchange. But all these effects [2] are proportional to the square of the mixing parameters. On the other hand the physical observables associated with $Z$ decay into third family fermions do receive corrections to linear order in the mixing parameters. It is appropriate to consider these latter effects in light of the new data from $Z$ factories.

The vector and axial couplings of the $X$ boson to members of the third family will be denoted by $g_{X v}^{f}$ and $g_{X a}^{f}$ with $f=t, b, \nu_{\tau}, \tau$. Other fermions (but not light fermions) may also carry $X$ charge, and when summed over all such fermions the $X$ charges are anomaly free and orthogonal to standard model hypercharges. The

\footnotetext{
${ }^{2}$ We discuss fermion mass mixing effects at the end.
} 
standard model $Z$ couplings will be denoted by $g_{v}^{f}$ and $g_{a}^{f}$.

The mixing effects are described by the following Lagrangian quadratic in the $A$, $Z$, and $X$ fields. Primes are attached to indicate that these fields, which have the couplings to matter just described, do not yet have conventional kinetic or diagonal mass terms.

$$
\begin{aligned}
\mathcal{L}_{A Z X}= & -\frac{1}{4} A^{\prime}{ }_{\mu \nu} A^{\prime \mu \nu}-\frac{1}{4} Z^{\prime}{ }_{\mu \nu} Z^{\prime \mu \nu}-\frac{1}{4} X^{\prime}{ }_{\mu \nu} X^{\prime \mu \nu} \\
& +\frac{1}{2} m_{Z}^{2} Z^{\prime}{ }_{\mu} Z^{\prime \mu}+\frac{1}{2} m_{X}^{2} X^{\prime}{ }_{\mu} X^{\prime \mu} \\
& +x m_{Z}^{2} Z^{\prime}{ }_{\mu} X^{\prime \mu}-y \frac{1}{2} Z^{\prime}{ }_{\mu \nu} X^{\prime \mu \nu}-w \frac{1}{2} A^{\prime}{ }_{\mu \nu} X^{\prime \mu \nu}
\end{aligned}
$$

We perform a transformation to unprimed fields which recovers conventional kinetic terms and a diagonal mass matrix. The full result to second order in the mixing parameters $x, y$, and $w$ is given in [2].

The relation of interest here is

$$
X^{\prime}{ }_{\mu}=X_{\mu}+(y-x) \frac{m_{Z}^{2}}{m_{X}^{2}} Z_{\mu}
$$

where we have kept only the first order terms in the mixing parameters and assumed that $m_{X}^{2} \gg m_{Z}^{2}$. When this substitution is made in the full Lagrangian we find that the $Z$ couplings to the third family are shifted by amounts

$$
\delta g_{v, a}^{f}=(y-x) \frac{m_{Z}^{2}}{m_{X}^{2}} g_{X v, a}^{f} .
$$

The best constraints on these couplings come from the partial decay widths, $\Gamma_{f}$, and the asymmetry parameters,

$$
A_{f}=\frac{2 g_{v}^{f 2} g_{a}^{f 2}}{g_{v}^{f 2}+g_{a}^{f 2}} .
$$

They are shifted by amounts

$$
\begin{gathered}
\frac{\delta \Gamma_{f}}{\Gamma_{f}}=\frac{2\left(g_{v}^{f} \delta g_{v}^{f}+g_{a}^{f} \delta g_{a}^{f}\right)}{g_{v}^{f 2}+g_{a}^{f 2}}, \\
\frac{\delta A_{f}}{A_{f}}=\frac{g_{a}^{f^{2}}-g_{v}^{f^{2}}}{g_{a}^{f^{2}}+g_{v}^{f^{2}}}\left(\frac{\delta g_{v}^{f}}{g_{v}^{f}}-\frac{\delta g_{a}^{f}}{g_{a}^{f}}\right) .
\end{gathered}
$$




\begin{tabular}{|c|l|l|}
\hline & Measurement & $X$ boson \\
\hline$\delta \Gamma_{b} / \Gamma_{b}$ & $\begin{array}{r}a)+0.031 \pm 0.011 \\
+0.030 \pm 0.014\end{array}$ & +0.021 \\
\hline$\delta \Gamma_{\nu_{\tau}} / \Gamma_{\nu_{\tau}}$ & $b)-0.014 \pm 0.023$ & -0.015 \\
\hline$\delta \Gamma_{\tau} / \Gamma_{\tau}$ & $c)+0.002 \pm 0.005$ & +0.0022 \\
\hline$\delta A_{\tau} / A_{\tau}$ & $d)+0.31 \pm 0.13$ & +0.21 \\
\hline$\delta A_{b} / A_{b}$ & $e)-0.02 \pm 0.16$ & -0.0054 \\
\hline
\end{tabular}

Table 1: These shifts are due to universality breaking effects only, and do not include effects already contained in the standard model.

The vector $Z$ coupling to $\tau$ is small, $g_{v}^{\tau} \approx 0.07 g_{a}^{\tau}$, and thus $\Gamma_{\tau}$ is really only sensitive to a shift in $g_{a}^{\tau}$. $\Gamma_{\tau}$ has been found to be consistent with lepton universality to a fraction of a percent, thus making $g_{a}^{\tau}$ the most constrained of the third family $Z$ couplings. If we wish this not to constrain the $Z-X$ mixing then we must ensure that the $X$ boson has only vector couplings to the $\tau$.

To proceed further we will consider an explicit model in which the $X$ charges of the third family are the following.

$$
\begin{aligned}
& g_{X v}^{t, b}=0 \quad, \quad g_{X a}^{t, b}=g_{X} \\
& g_{X v}^{\tau}=g_{X} \quad, \quad g_{X a}^{\tau}=0 \\
& g_{X \nu}^{\nu_{\tau}} \equiv g_{X v}^{\nu_{\tau}}+g_{X \tau}^{\nu_{\tau}}=g_{X}
\end{aligned}
$$

The model we have in mind is a recently proposed dynamical model for the large top mass, in which the quarks are required to have axial couplings to the $X$ boson. [3] We will find that the source of $Z$ - $X$ mixing in the model also relies on the axial $X$ couplings to quarks.

We first present the results. In Table 1 we compare the measured shifts of five observables to the expected shifts due to the $X$ boson. The relative sizes and relative signs of the expected shifts are completely determined, given the $X$ charges in (7). The model then provides the additional input to determine the overall sign and to allow an estimate of the overall magnitude of the shifts.

In the measurements we isolate those departures from universality beyond those already contained in the standard model. We are not interested in univerality pre- 
serving corrections such as oblique corrections from new physics. The measured shifts in Table 1 do not include oblique corrections because we are careful to extract the shifts from observables insensitive to oblique corrections. In particular, the values in the table labeled $a$ ) to $e$ ) were obtained as follows.

a) We use $\Gamma_{b} / \Gamma_{\text {had }}=\Gamma_{b} /\left(\Gamma_{b}+\Gamma_{\text {had } \neq b}\right)$ and compare its value, from 《⿴囗十 and [5] respectively, to the standard model value of 0.2158 for a top mass of $175 \mathrm{GeV}$.

b) We extract the value of $\Gamma_{\text {inv }} / \Gamma_{\ell}=\left(\Gamma_{\nu_{e}}+\Gamma_{\nu_{\mu}}+\Gamma_{\nu_{\tau}}\right) / \Gamma_{\ell}$ from [4] using the near $e-\mu-\tau$ universality for $\Gamma_{\ell}$, and compare to a standard model value of 5.976 .

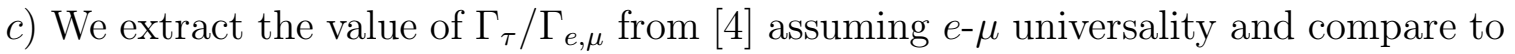
unity.

d) The ratios $A_{F B}^{0 \tau} / A_{F B}^{0 e, \mu}$ from forward-backward measurements and $3 P_{\tau} / 4 P_{\tau}^{F B}$ from $\tau$ polarization measurements are both equal to $A_{\tau} / A_{e}$ assuming $e-\mu$ universality. We obtain these two ratios from [5] and then average them and compare to unity. This departure from unity appears to be as significant as the more publicized $\delta \Gamma_{b} / \Gamma_{b}$. (Caution: SLD reports a value for $A_{e}$ inconsistent with and larger than the LEP values.)

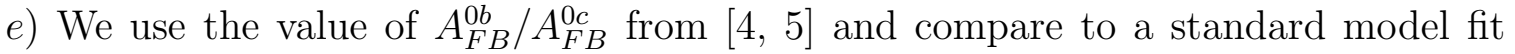
value of 1.4 [5]. The errors, especially in $A_{F B}^{0 c}$, make this uninteresting.

The $X$ boson also leads to a decrease in the value of $\alpha_{s}\left(m_{Z}\right)$ extracted from the measurement of $R_{\ell}=\Gamma_{\text {had }} / \Gamma_{\ell}$. This is because $R_{\ell}$ remains the same if the increase in $\Gamma_{b}$ is compensated by a decrease in the QCD corrections to $\Gamma_{\text {had }}$. Using the $\alpha_{s}$ dependence of $R_{\ell}$ in [6] we find that the shift is $\delta \alpha_{s}\left(m_{Z}\right)=-0.014$. Shifting the value of $\alpha_{s}\left(m_{Z}\right)$ in $[$, 河 by this amount gives

$$
\alpha_{s}\left(m_{Z}\right)=0.110 \pm 0.007 \text {. }
$$

This is typical of values of $\alpha_{s}\left(m_{Z}\right)$ obtained from deep inelastic scattering and heavy quarkonia decays, and is lower than values from jet studies.[7]

We now briefly describe the model [3] and derive our results. The model contains a fourth family, and members of the third and fourth families are composed from two "families" of fermions $f$ and $\underline{f}$. The massive $X$ boson, which appears when a nonabelian hypercolor gauge group breaks, couples with a vector charge of $+g_{X}$ to 
all members of the $f$ family and with a vector charge of $-g_{X}$ to all member of the $\underline{f}$ family. Each of these families has standard $S U(3) \times S U(2) \times U(1)$ quantum numbers. But these fields are not the mass eigenstates. The mechanism producing a large top mass requires that the fourth family quark mass eigenstates $t^{\prime}$ and $b^{\prime}$ correspond to Dirac spinors of the form $\left[\underline{f}_{L}, f_{R}\right]$. The $t^{\prime}$ and $b^{\prime}$ are nearly degenerate with masses close to a TeV. The $t$ and $b$ quarks correspond to $\left[f_{L}, \underline{f}_{R}\right]$, which then implies that the $X$ boson couples with the same axial coupling to the $t$ and $b$ quarks.

We will see that this leads to a positive shift in $\Gamma_{b}$. This prediction is to be compared with the negative shift found in the simplest extended technicolor theories. [1] The analog of this negative ETC contribution does not exist in our model since there is no gauge boson which causes a transition between a $b$ and a more massive fermion other than the $t$. At one loop, the only nonstandard correction to the $Z b \bar{b}$ vertex is due to $Z-X$ mixing.

The mechanism by which the top receives a large mass need not also be occurring in the lepton sector, and thus the lepton mass eigenstates were not determined in [3]. What is happening in the lepton sector is uncertain, and thus we shall simply assume that the dynamics of the model is such that the leptonic $\underline{f}$ fields describe the fourth family leptons (with masses somewhat less than the $t^{\prime}$ and $b^{\prime}$ masses). The $\tau$ and the $\nu_{\tau}$ are then described by leptonic $f$ fields; this implies for example the vector $X$ coupling to the $\tau$. Notice that the leptonic $X$ charges would change sign if the dynamics of the model led to the reverse identification of the $\underline{f}$ and $f$ fields. This would change the overall sign of the shifts in the leptonic observables.

The universality breaking effects are ultimately caused by $X$ boson exchange, and thus we need the value of $g_{X}^{2} / m_{X}^{2}$. The model provides an estimate since the main source of mass for both the $X$ and $Z$ bosons is the dynamical $t^{\prime}$ and $b^{\prime}$ masses. The ratio of the $X$ and $Z$ masses is determined by the ratio of their respective axial couplings to these quarks.

$$
\frac{g_{X}^{2}}{m_{X}^{2}}=\frac{\left(\frac{e}{4 c s}\right)^{2}}{m_{Z}^{2}}
$$

The $Z$ and to a lesser extent the $X$ also receive a small contribution to their mass from the fourth family leptons. This would reduce $m_{X} / m_{Z}$ and thus slightly increase our estimate of $g_{X}^{2} / m_{X}^{2} \cdot g_{X}$ must be large enough to ensure that $m_{X}^{2} / m_{Z}^{2} \gg 1$, as 
we have previously assumed. This is reasonable since the $X$ boson emerges from a strongly interacting hypercolor interaction.

Because of the $X$ couplings a quark loop can produce $Z$ - $X$ mass mixing only if there is isospin violating quark masses. The main motivation for the model was to show how $t^{\prime}$ and $b^{\prime}$ can remain almost degenerate and thus produce little contribution to $\Delta \rho$. Similarly, the $t^{\prime}$ and $b^{\prime}$ contributions to $Z$ - $X$ mass mixing will largely cancel. The $\tau^{\prime}$ loop contribution nearly vanishes since the $Z$ and $X$ have essentially axial and vector $\tau^{\prime}$ couplings respectively. The massive $\nu_{\tau^{\prime}}$ could contribute, but its mass is also constrained by $\Delta \rho$. Thus it is fair to assume that the $t$ loop is the main source of $Z-X$ mass mixing.

We may determine the mixing parameter $x$ as defined in (四) by taking the ratio of the $Z$ - $X$ mixing diagram (a $t$ loop) to the $Z$ mass diagram (a $q^{\prime}$ loop). These loop diagrams are convergent and are dependent on the momentum-dependent dynamical mass functions for the $t$ and $q^{\prime}$. Neither of these masses arise from a simple ETC boson exchange, and thus both mass functions are expected to fall with increasing momentum more quickly than quark masses in conventional ETC theories. If one mass function was just the scaled version of the other mass function then the result for $x$ would be

$$
x=\frac{\frac{e}{4 c s} g_{X} m_{t}{ }^{2}}{\left(\frac{e}{4 c s}\right)^{2} 2 m_{q^{\prime}}{ }^{2}} .
$$

The factor of 2 is for the two flavors $t^{\prime}$ and $b^{\prime}$. We will absorb the uncertainty in the mass functions into the uncertainty in the value of $m_{q^{\prime}}$. In [3] we estimated $m_{q^{\prime}} \approx 1$ $\mathrm{TeV}$.

The mixing parameter $y$ receives contributions from a $b$ loop and a $\nu_{\tau}$ loop. These loops are log divergent and they are cutoff by the $t$ mass and $\nu_{\tau^{\prime}}$ mass respectively. By setting a logarithm equal to unity we estimate

$$
y \approx \frac{e}{4 c s} \frac{g_{X}}{6 \pi^{2}} .
$$

This turns out to be less than $4 \%$ of $x$, and thus we will simply ignore $y$. 
By combining the results in (3), (7), (9), and (10) we find

$$
\begin{gathered}
\delta g_{v}^{t, b}=0 \quad, \quad \delta g_{a}^{t, b}=Y \\
\delta g_{v}^{\tau}=Y \quad, \quad \delta g_{a}^{\tau}=0 \\
\delta g_{L}^{\nu}=Y
\end{gathered}
$$

with

$$
Y=-\frac{e}{8 c s}\left(\frac{m_{t}}{m_{q^{\prime}}}\right)^{2} .
$$

Notice that we have not needed to determine $m_{X}$ or $g_{X}$ separately; and although the sign of $x$ depends on the sign of $g_{X}$, the final result does not. By inserting (12) along with $m_{t}=175 \mathrm{GeV}, m_{q^{\prime}}=1 \mathrm{TeV}$, and $s^{2}=.232$ into (5) and (6) gives the shifts in Table 1. Also, by combining our results with those of [2] we have confirmed that the $Z$ - $X$ mixing has a negligible effect on oblique parameters $S, T$, and $U$ and neutral current processes. Among these, the largest change is a 0.03 increase in $T$.

Finally, an $X$ boson having nonuniversal flavor couplings is an obvious source of flavor changing neutral currents (FCNCs) among light quarks, because of the fermion mass mixing which must occur between families. For example an $X$ boson exchange produces an operator $\bar{t} \gamma_{\mu} \gamma_{5} t \bar{t} \gamma^{\mu} \gamma_{5} t$. When expressed in terms of the quark mass eigenstates there may be a contribution to the $\Delta C=2$ operator $\bar{c} \gamma_{\mu} \gamma_{5} u \bar{c} \gamma^{\mu} \gamma_{5} u$. The size of this flavor changing effect depends on how the mass mixing arises in the model. If, as suggested in [3], all mass mixing between families arises in the up sector then the resulting $D^{0}-\bar{D}^{0}$ mixing will involve a factor of order $\left|V_{u b} V_{c b}\right|^{2}$ where $V$ is the KM matrix. This provides more than adequate suppression. Mass mixing would only be transmitted to the down sector via additional weak effects, and this would keep the contributions to $K^{0}-\bar{K}^{0}$ and $B^{0}-\bar{B}^{0}$ mixing at a safe level. This type of scenario has been described [8] in the context of the usual FCNC problem of technicolor theories.

The $X$ boson we have described causes a distinctive pattern of universality breaking corrections. The comparison with the data in Table 1 is tantalizing, and we hope that this motivates further interest in the search for new flavor physics in precision experiments. 


\section{Acknowledgments}

I thank D. Bailey for discussions. This research was supported in part by the Natural Sciences and Engineering Research Council of Canada.

\section{References}

[1] R.S. Chivukula, B. Selipsky, E.H. Simmons, Phys. Rev. Lett. 69 (1992) 575; R.S. Chivukula, E. Gates, E.H. Simmons, and J. Terning, Phys. Lett. B311 (1993) 157; R.S. Chivukula, E.H. Simmons, J. Terning, Boston University preprint, BUHEP-94-8, hep-ph/9404209, April 1994.

[2] B. Holdom, Phys. Lett. B259 (1991) 329.

[3] B. Holdom, University of Toronto, UTPT-94-18, hep-ph/9407244, June 1994 (to appear in Phys. Lett. B).

[4] R. Miquel, CERN preprint, CERN-PPE/94-70, May 1994.

[5] B. Pietrzyk, Laboratoire de Physique des Particules preprint, LAPP-EXP-94.07, hep-ex/9406001, May 1994; B. Jacobsen, CERN preprint, CERN-PPE/94-97, June 1994.

[6] T. Hebbeker, M. Martinez, G. Passarino, G. Quast, CERN-PPE/94-44, March 1994.

[7] eg. S. Bethke, ICHEP 92, Dallas, HEP 1992, p81 (QCD161:I41:1992).

[8] B. Holdom, Phys. Lett. B143 (1984) 227; A. Hadeed and B. Holdom, Phys. Lett. B159 (1985) 379. 